\title{
Sea Surface Characterization using Dual Polarized GNSS Reception System
}

\author{
Ankit Regmi $^{1}$, Aarno Pärssinen ${ }^{1}$, Markus Berg ${ }^{1}$ \\ Centre for Wireless Communications ${ }^{1}$, University of Oulu, Oulu, Finland, ankit.regmi@oulu.fi
}

\begin{abstract}
GNSS signal reception using Dual Circular Polarized method has been proposed to simultaneously record direct and reflected signals from the sea surface. Dual circular polarized reception (DCPR) system gives the opportunity to exploit the polarization change of the incident signal after reflection. This paper reports received signal characteristics of various GPS satellites for bare sea conditions. The received signals from various satellites are compared and are analyzed statistically to characterize the sea-state. The reflected left-hand circular polarized (LHCP) signal is used to analyze the scattering characteristics of sea surface. The statistics of LHCP signal give strong correlation with the wind speed over the sea and can be used to characterize the sea-state.
\end{abstract}

Index Terms-GNSS-R, multipath, dual CP antenna, reflection, wind speed.

\section{INTRODUCTION}

Global Navigation Satellite System (GNSS) signals are freely available and can be received with compatible receivers, globally. GNSS is mainly used for positioning and navigational purpose. The era of GNSS-Reflectometry (GNSS-R) began two-decades ago, when an aircraft locked on to GNSS signals reflected off the sea-surface, resulting in false positioning information. However, further analysis of the data gave the altimetry information of the aircraft above sea. [1], [2]. Since then, GNSS-R has been very active in research in environment remote sensing. It has been used in application such as seastate determination, wind velocity over the ocean, extraction of snow and ice-thickness, soil moisture and vegetation determination, multipath environment characterization, etc [3]-[5].

Non-line-of-sight (NLOS) signal or multipath component are considered unwanted parameter in GNSS as it degrades the positioning accuracy. However, they are a figure of merit in the field of GNSS-R. Over the year, GNSS-R has been applied using techniques, such as bistatic radar and interferometric reflectometry. As bistatic radar, the delayed GNSS reflected signal gives the distance information to the target and waveform gives the characteristics of the target. In GNSSInterferometric Reflectometry (GNSS-IR), the direct and reflected GNSS signal add with continuously changing phase due to excess path length travelled by the reflected signal which results in interference pattern [6], [7]. The frequency of the interference pattern is used to estimate the height of the antenna phase center above the horizontal reflecting surface and the reflection coefficient can be extracted using the interference pattern technique (IPT) [8]. Dual circular polarized reception (DCPR) has been used to extract surface reflection coefficients, sea-level estimation, as well as NLOS detection and mitigation [9]-[11].

In this study, DCPR technique is used to characterize the sea-state. This method exploits the polarization change of the incident right-hand circular polarized (RHCP) signal into left hand circular polarized (LHCP) after signal reflection from sea-surface, and thus requires simultaneous recording of multipath fading RHCP signal and reflected LHCP signal.

This paper is arranged in the following order. Section II gives fundamentals on GNSS-IR and reception using DCPR system. Section III describes the measurement system and scenario. The measurement results and analysis are presented in Section IV. Conclusion and future prospects are discussed in Section V.

\section{GNSS-INTERFEROMETRIC REFLECTOMETRY AND DUAL CIRCUlar POLARIZED RECEPTION}

GNSS satellites transmit RHCP signals at different frequency bands depending on the various satellite constellation systems. In this work, GPS satellite system transmitting at L1 band with centre frequency $1.575 \mathrm{GHz}$ is used. The received LOS or incident signal is RHCP, however, the nature of reflected signal depends on the dielectric properties of surface and frequency of incident signal. The incident RHCP signal undergoes 180 degree phase reversal after reflection from a smooth surface. This phase reversal takes place after the Brewster angle and the resultant signal will be LHCP. This physical phenomenon gives the opportunity for DCPR system to simultaneously receive and process direct RHCP and reflected RHCP plus LHCP signals.

\section{A. Propagation}

The received signal is the combination of incident RHCP and reflected RHCP plus LHCP signal. The reflected RHCP becomes more and more elliptic as it approaches the Brewster angle. At Brewster angle, the vertical component of electric field becomes zero resulting in linearly polarized wave. As the elevation angle becomes greater than the Brewster angle, the wave becomes left-hand elliptically polarized and gradually the ellipicity decreases resulting in LHCP wave. [12]

\section{B. $D C P R$}

The RHCP received signal is the addition of direct and reflected signal with phase difference equal to excess path length travelled by reflected signal. This causes the signals to add constructively and destructively, at same and opposite 
phases respectively, resulting in an interference pattern. The frequency of this interference pattern, also known as multipath frequency, is the function of carrier wavelength, angle of elevation, permittivity of reflecting medium, vertical height of antenna above the reflecting surface and polarization of the signal. The variation in antenna height changes the frequency of the interference signal [13]. Thus, this variation of height or multipath frequency can be used to retrieve parameters such as sea surface height, snow-thickness, altimetry, etc. and are reported in [6], [7]. However, a DCPR system also has the capability to distinctively receive the reflected LHCP signal. LHCP signal is mainly the function of surface reflection coefficient i.e. it comprises of reflected or scattered components. Analysis of LHCP signal provides more detailed information about the surface characteristics.

Dual circular polarized (CP) antenna is the fundamental element to perform DCPR. For a dual CP antenna, the key design parameters are radiation pattern, cross-polarization discrimination (XPD), port isolation and minimum phase center variations $(\mathrm{PCV})$. For simultaneous reception of right and left hand signals, the XPD must be large enough to suppress the cross-polarized components. XPD of the antenna acts as a polarization filter to suppress unwanted cross-polarized component. In GNSS-R the reflection coefficient is calculated considering the cross-polarized component and is known as coupled-reflection coefficient [13]. The classification of received signal taking into account the XPD of the antenna can be found in [4].

\section{Measurement System and Scenario}

Coastal sea surface measurements were performed at Nallikari Beach in Oulu, Finland. The coordinates for measurement site were $65^{\circ} 1^{\prime} 50.88^{\prime \prime} \mathrm{N}$ and $25^{\circ} 24^{\prime} 38.52^{\prime \prime} \mathrm{E}$. The measurement duration was approx. 4 hours. The average temperature was $6^{\circ} \mathrm{C}$ during the measurement period. The measurement setup and scenario is shown in Fig. 1. The satellite passage, trajectory including elevation and azimuth angle are shown in Table 1.

TABLE I

SATELLITE TRAJECTORIES

\begin{tabular}{|c|c|c|c|c|}
\hline UTC & PRN & Elevation & Azimuth & Trajectory \\
\hline 10:09:30-11:01:58 & 31 & $5-24$ & $313-310$ & Rising \\
\hline 10:16:42-11:11:33 & 29 & $1-26$ & $215-219$ & Rising \\
\hline 10:27:11-12.09.10 & 32 & $31-1$ & $284-251$ & Setting \\
\hline 10:58:53-12:41:20 & 14 & $31-1$ & $290-256$ & Setting \\
\hline 12:01:40-13:14:32 & 26 & $1-31$ & $293-295$ & Rising \\
\hline
\end{tabular}

A differentially-fed dual CP GNSS antenna with high isolation, 27-29 dB between the left and right hand ports was used. The XPD of the antenna is greater than $25 \mathrm{~dB}$ at boresight and has symmetrical azimuth pattern. Details of the antenna can be found in [14]. The antenna was mounted horizontally on a tripod to ensure reception at equal gains for direct and reflected signals. Two identical u-blox GNSS evaluation kits were connected to the right and left hand ports of the antenna

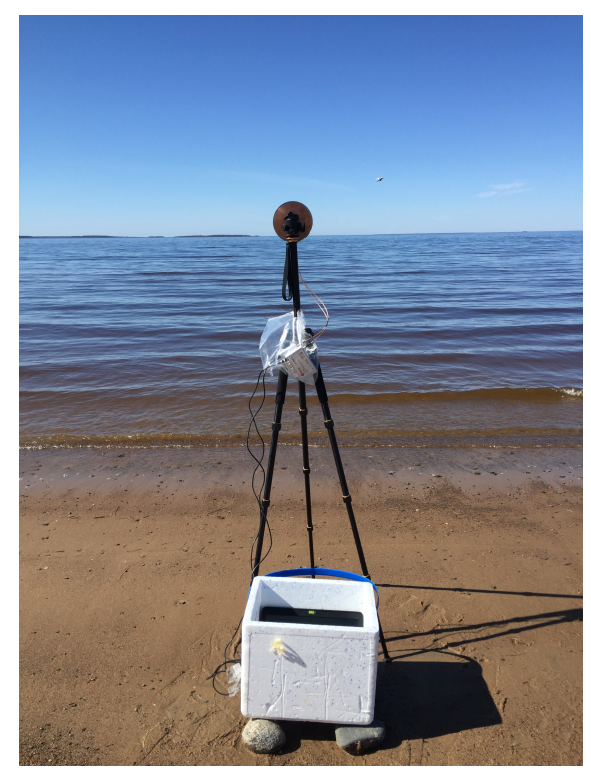

Fig. 1. Sea Surface Measurement

to ensure simultaneous reception of RHCP and LHCP signals. The receiver sampling frequency was set to $1 \mathrm{~Hz}$.

\section{A. Fresnel Zones}

Most of the reflected power comes from the first Fresnel zone (FFZ) if the reflecting surface is homogeneous and large compared to the FFZ. The size of the Fresnel ellipse becomes smaller with increasing elevation angle. The FFZ is the function of satellite elevation and azimuth angle and vertical height of the antenna considering specular reflection [15]. The specular point of reflection is the centre of the Fresnel ellipse and can be calculated using Snell's law of reflection. The FFZs for used satellites with respect to the elevation and azimuth variation is shown in Fig. 2.

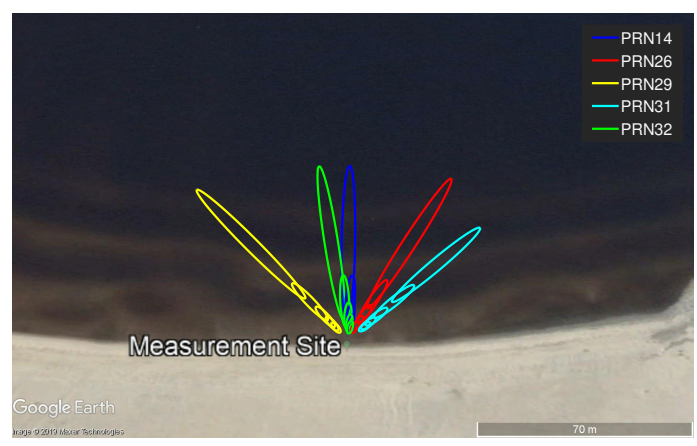

Fig. 2. FFZ w.r.t. satellite elevation and azimuth variation

Elevation angles from $5^{\circ}$ to $25^{\circ}$ at $5^{\circ}$ interval and the corresponding azimuth angles are shown in Fig. 2, where the advantage of GNSS-R for environment remote sensing is also seen clearly. The satellite footprints distributed over azimuth extend to significantly wide areas, even for small antenna height. This gives the opportunity to scan larger areas and 
get more detailed information about the sea surface compared to locally placed sensors.

\section{ReCEIVEd Signal Characteristics}

RHCP and LHCP signals reflected from the sea surface were recorded simultaneously using DCPR system. The satellite signals for analysis were chosen on the basis of most suitable elevation and azimuth angles with respect to the antenna orientation. GPS satellites PRN14, PRN26, PRN29, PRN31, PRN32 were chosen for analysis. The effect of LOS signal has been removed by using the radiation pattern of the antenna [4]. The detrended carrier-to-noise density ratio $(\delta \mathrm{C} / \mathrm{N} 0)$ as a function of elevation angle for RHCP and LHCP received response are shown in Fig. 3.
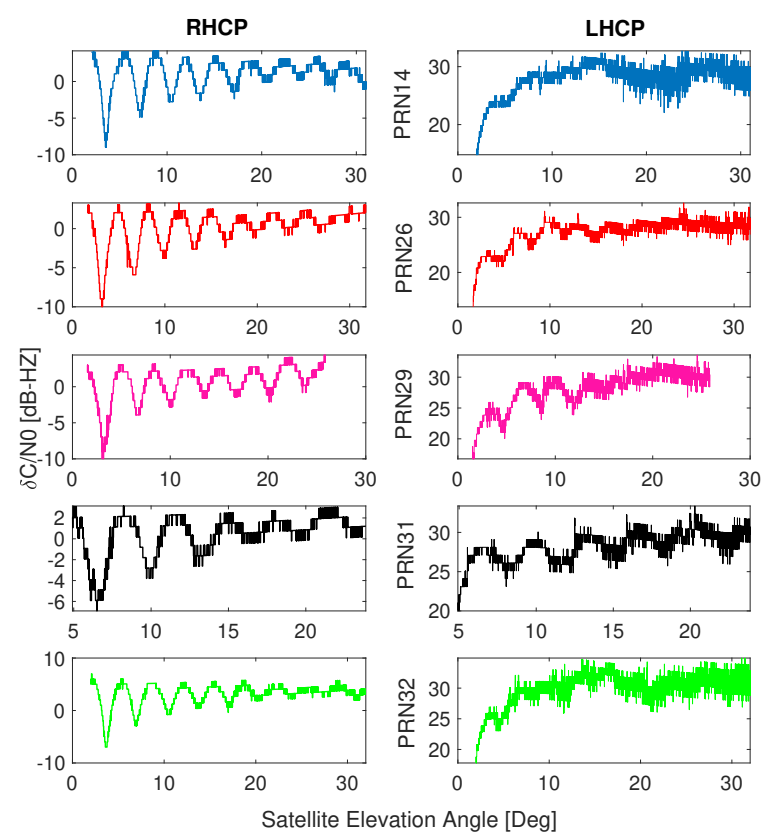

Fig. 3. RHCP and LHCP $\delta$ C/No for PRN14, PRN26, PRN29, PRN31, PRN32, respectively vs Elevation Angle

The RHCP interference pattern is due to the satellite movement in the elevation plane and this is clearly observed for all the satellites. Satellite has approximately constant angular velocity with respect to the center of the Earth [16]. As the satellite moves more in the azimuth its movement in the elevation becomes slower. Therefore, the azimuth movement of the satellites has been compensated for all satellite data. The multipath frequency is approximately constant at lower elevation angles, but changes faster at higher elevation angles. Therefore, the elevation angles have been limited to region where clear interference patterns are visible. The multipath interference frequency is given in by [17],

$$
f_{m u l}=\frac{1}{2 \pi} \frac{\delta \phi}{\delta \sin \theta}
$$

where,

$$
\phi=\frac{(4 \pi h \sin \theta)}{\lambda}
$$

Here, $\phi$ represents the phase change due to excess path length between direct and reflected signals, $h$ is the antenna phase center height above the reflecting surface, $\theta$ is the satellite elevation angle, $\lambda$ is the carrier wavelength.

LHCP is the function of elevation angle and the reflection coefficient of the sea-surface. However, in Fig. 3 at lower elevation angles a clear interference pattern can be seen for all satellites. This is due to the nature of the signal being RHCP before the Brewster angle. Hence, even with high XPD, crosspolarized product is clearly visible at lower elevation angle. However, the LHCP signal dominates after the Brewster angle and the interference pattern disappears. LHCP data contains specularly reflected and scattered signals from the sea-surface.

\section{A. Spectrum Analysis}

The frequency content of the received signal gives valuable information of the sea-state. Spectrum of RHCP time series can be directly presented as the function of antenna height above the horizontal reflecting surface. Hence, any fluctuation in the sea-level results in different antenna height. LombScargle Periodogram (LSP) is used to perform Fast Fourier analysis of the time series for all satellites [18]. RHCP signal spectrum as a function of antenna height above sea-surface are shown in Fig. 4.

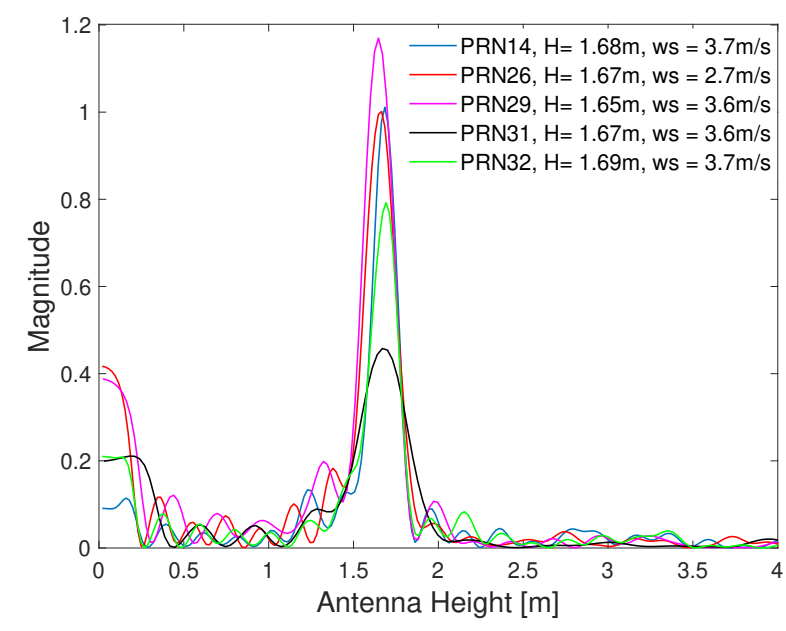

Fig. 4. LSP of RHCP data as the function of Antenna Height

The average wind speed for the duration of each satellite pass is also mentioned in Fig. 4. The wind speed data had been extracted from Finnish Meteorological Institute's (FMI) marine observation data. The altimetry information and wind speed shows a stable sea state, however, clear correlation between wind speed and antenna height can be seen for PRN 14 and PRN 32. However, more information is required to validate this observation. Small magnitude and wide peak for PRN 31 corresponds to the azimuth difference between antenna and the satellite.

The LHCP spectrum is shown in Fig. 5. The DC (zero frequency) component is not included in the figure. The peaks on the left signifies the reflection from the surface. For a 


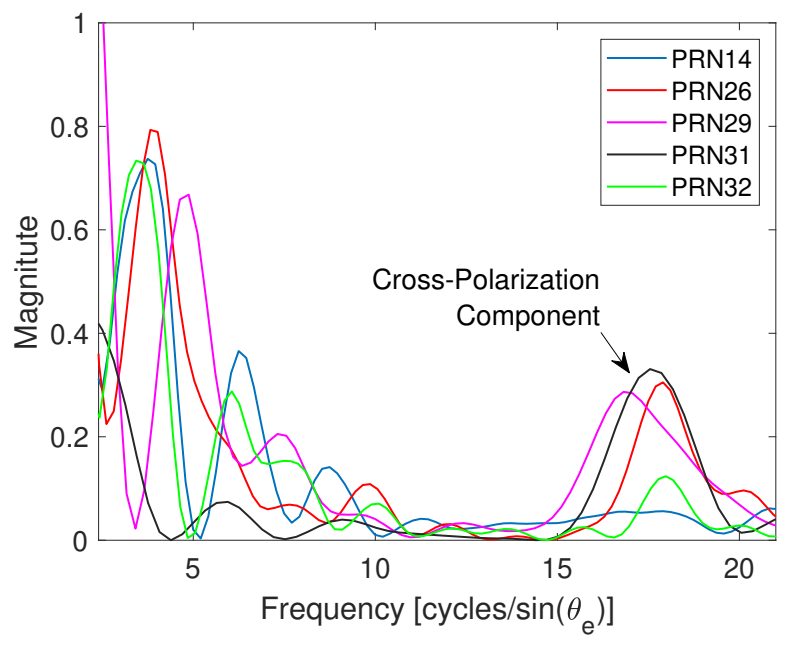

Fig. 5. LSP of LHCP data as the function of reflector height

smooth surface where specular reflection occurs, the single peak on the left dominates the spectrum. Multiple peaks will be visible in case of many scattered components signifying rough surface. PRN26 and PRN 29 have single dominant peaks, whereas for PRN14 and PRN32, multiple peaks are visible, signifying rougher sea conditions.

The peaks on the right side indicates the cross-polarized (XP) right-hand component of the signal. The XP peak also gives valuable information on roughness of the surface. As seen in Fig. 5, for smooth surface, the spectrum of PRN26, PRN29 and PRN31 gives clear XP peaks directly related to antenna height above surface, whereas for PRN32, the XP peak is attenuated significantly and for PRN 14 completely disappears signifying more scattering and rougher surface.

\section{B. Statistical Analysis}

RHCP altimetry information is not enough to characterize sea-surface roughness. LHCP signal mainly comprises of reflected and scattered component and is more effective in analysing sea-state (roughness). Rough sea has direct correlation with the LHCP signal. As can be seen in Fig. 3, for LHCP, some satellite data are more noisy than others. More noise corresponds to more surface roughness and can be directly linked to the wind speed over the sea. Matlab application, Signal Analyzer is used to extract the noise statistics of the received LHCP signal for all satellites. The LHCP signal was passed through a high-pass filter with passband frequency $0.06 \mathrm{~Hz}$ to extract the noise in the signal greater than the antenna height. Noise distribution for all the satellites PRN 14, 26, 29, 31, 32 are shown in Fig. 6, respectively.

The roughness of the sea surface is a function of satellite elevation angle. Also, the size of FFZ and the specular reflection point vary with the change in elevation angle as shown in Fig. 2. At lower angle of elevation the specular reflection dominates. Whereas increased scattering is observed at higher elevation angle that results in reduced magnitude

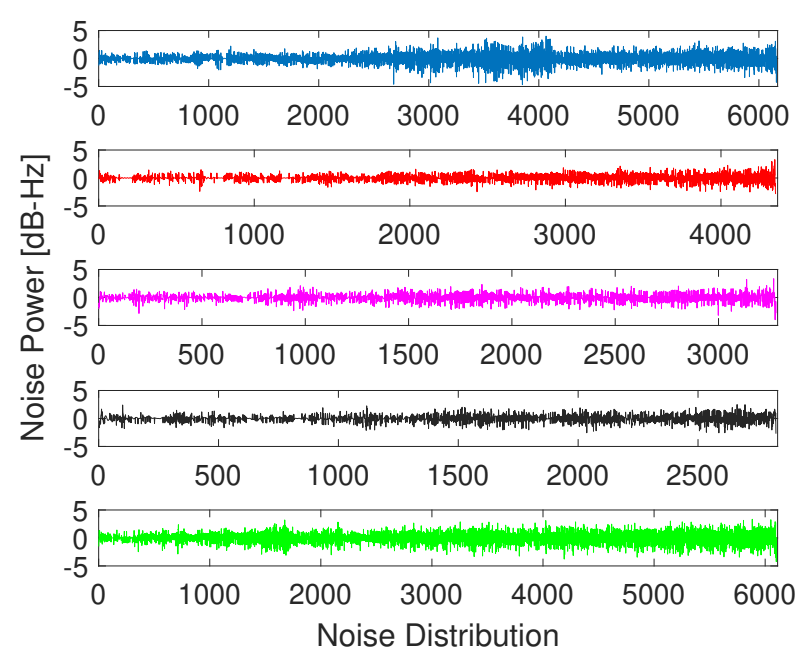

Fig. 6. Noise Power vs Noise Distribution

of specular reflected components. This phenomenon can be observed for all satellites in Fig. 6. Standard deviation (STD) of noise was calculated using data from Fig. 6. STD for every 10 minutes duration of the data was performed. The noise STD for all satellites are compared with 10 minutes wind speed data given by FMI and are shown in Fig. 7.

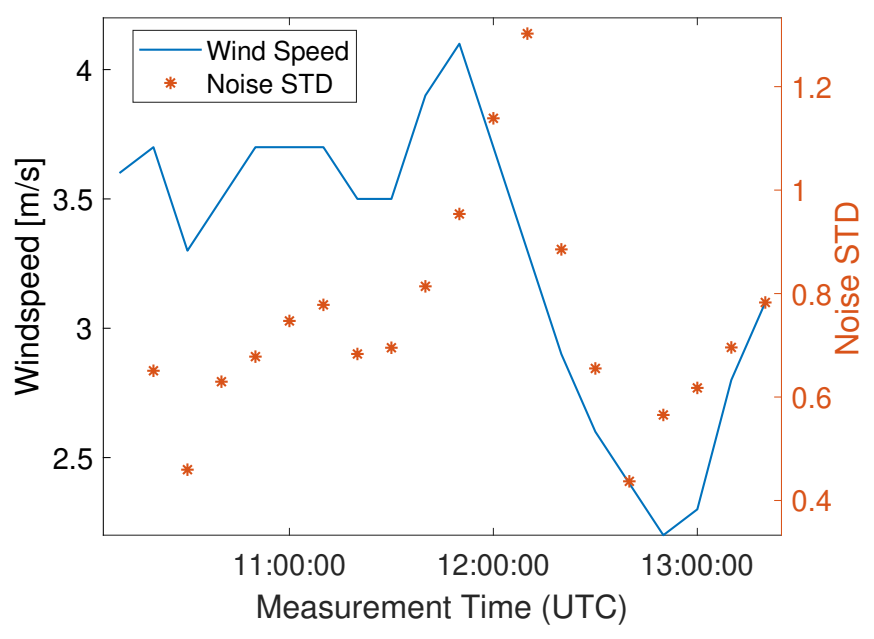

Fig. 7. Correlation between windspeed and LHCP Noise STD vs Measurement Time

A clear correlation can be seen between wind speed over sea and noise statistics of LHCP reflected signal for high and low wind speeds. Hence, scattered signal from the sea provides key information about the sea-state. The purpose of Fig. 7 is to give the reader a clear view of the correlation between wind speed and noise STD of reflected signal. Formulating a windspeed determination model based on the analysis presented here is beyond the scope of this paper. More measurement data for different wind speeds is required prior to the LHCP noise characteristics can be used to generate a model for wind speed. 


\section{CONCLUSion}

A low cost DCPR system was proposed to perform GNSS-R measurement to characterize sea-state using C/N0 data. GPS satellites with different azimuth angle covered wide area for sensing purpose. Spectrum and statistical analysis were performed to relate RH and LH signals with wind speed over the sea and roughness of the sea. RHCP spectrum analysis gave the antenna height above sea-surface. More information about sea-state was clearly visible in LHCP spectrum. Statistical analysis was performed to relate sea roughness, wind speed and reflected LHCP signal. STD of reflected LHCP noise was compared with the wind speed observation from FMI. A clear correlation between noise and wind speed was observed. However, more measurements has to be performed for different seastate to statistically model sea surface roughness. This paper also highlights the advantages of using DCPR system over single RHCP antenna system in GNSS-R configuration.

\section{ACKNOWLEDGMENT}

This work was supported in part by the Academy of Finland 6Genesis Flagship (grant no. 318927). InfoTech Oulu is also acknowledged for financial support through doctoral training position.

\section{REFERENCES}

[1] M. Martin-Neira, "A passive reflectometry and interferometry system (paris): Application to ocean altimetry," ESA Journal, vol. 17, pp. 331$355,1993$.

[2] J. C. Auber, A. Bibaut, and J. M. Rigal, "Characterization of multipath on land and sea at GPS frequencies," in Proceedings of the 7th International Technical Meeting of the Satellite Division of The Institute of Navigation (ION GPS 1994), Salt Lake City, UT, September ,1994, pp. 1155-1171.

[3] K. M. Larson, E. D. Gutmann, V. U. Zavorotny, J. J. Braun, M. W. Williams, and F. G. Nievinski, "Can we measure snow depth with GPS receivers?" Geophysical Research Letters, vol. 36, no. 17, 2009.

[4] A. Regmi, M. Berg, and A. Pärssinen, "A method for ice thickness characterization using GNSS C/NO data," in Proceedings of 8th AsiaPacific Conference on Antennas and Propagation (APCAP), 2019, pp. $1-5$.

[5] N. Rodriguez-Alvarez, X. Bosch-Lluis, A. Camps, M. Vall-llossera, E. Valencia, J. F. Marchan-Hernandez, and I. Ramos-Perez, "Soil moisture retrieval using GNSS-R techniques: Experimental results over a bare soil field," IEEE Transactions on Geoscience and Remote Sensing, vol. 47, no. 11, pp. 3616-3624, Nov 2009.

[6] K. M. Larson and F. G. Nievinski, "GPS snow sensing: results from the earthscope plate boundary observatory," GPS Solutions, vol. 17, no. 1, pp. 41-52, Jan 2013.

[7] M. B. Rivas and M. Martin-Neira, "Coherent GPS reflections from the sea surface," IEEE Geoscience and Remote Sensing Letters, vol. 3, no. 1, pp. 28-31, Jan 2006.

[8] K. M. Larson, "GPS interferometric reflectometry: applications to surface soil moisture, snow depth, and vegetation water content in the western united states," Wiley Interdisciplinary Reviews: Water, vol. 3, no. 6, pp. 775-787, 2016.

[9] Y. Jia, P. Savi, D. Canone, and R. Notarpietro, "Estimation of surface characteristics using GNSS LH-reflected signals: Land versus water,' IEEE Journal of Selected Topics in Applied Earth Observations and Remote Sensing, vol. 9, no. 10, pp. 4752-4758, Oct 2016.

[10] M. Berg, R. U. R. Lighari, J. Kallankari, V. Majava, A. Pärssinen, and E. T. Salonen, "Polarization based measurement system for analysis of GNSS multipath signals," in 2016 10th European Conference on Antennas and Propagation (EuCAP), April 2016, pp. 1-4.
[11] A. Alonso Arroyo, A. Camps, A. Aguasca, G. F. Forte, A. Monerris, C. Rüdiger, J. P. Walker, H. Park, D. Pascual, and R. Onrubia, "Dualpolarization GNSS-R interference pattern technique for soil moisture mapping," IEEE Journal of Selected Topics in Applied Earth Observations and Remote Sensing, vol. 7, no. 5, pp. 1533-1544, May 2014.

[12] C. Balanis, Advanced Engineering Electromagnetics. Wiley, 1989.

[13] B. M. Hannah, "Modelling and simulation of GPS multipath propagation," Ph.D. dissertation, Queensland University of Technology, 2001.

[14] M. Berg, J. Chen, and A. Pärssinen, "Radiation characteristics of differentially-fed dual circularly polarized GNSS antenna," in 2019 13th European Conference on Antennas and Propagation (EuCAP), March 2019, pp. 1-5.

[15] C. Roesler and K. M. Larson, "Software tools for GNSS interferometric reflectometry (GNSS-IR)," GPS Solutions, vol. 22, no. 3, p. 80, Jun 2018.

[16] B. Parkinson, J. Spilker, and G. Elkaim, Global Positioning System (GPS). American Cancer Society, 2003.

[17] Y. Georgiadou and A. Kleusberg, "On carrier signal multipath effects in relative gps positioning," Manuscripta geodaetica, vol. 13, no. 3, pp. 172-179, 1988.

[18] K. M. Larson, J. S. Löfgren, and R. Haas, "Coastal sea level measurements using a single geodetic gps receiver," Advances in Space Research, vol. 51, no. 8, pp. $1301-1310,2013$. 